\title{
Microdistribution of plutonium in human skeleton. Should we change the ICRP model?
}

\author{
Sergei Romanov ${ }^{1, *}$, Ekaterina Lyovkina ${ }^{1}$, Sergei Sypko ${ }^{1}$, and Aleksandr Ephimov $^{1}$ \\ ${ }^{1}$ Southern Urals Biophysics Institute, FMBA of Russia
}

Skeleton is one of the major organs of plutonium deposition within the human body. It is well known that plutonium distribution in bone tissue is quite uneven. According to generally accepted model, main fraction of plutonium activity resides on endosteal bone surface and Haversian canal walls. Present study is devoted to analyzing plutonium microdistribution in the thoracic vertebra obtained from a former Mayak PA worker and preserved by SUBI Human Tissue Repository; the analysis was carried out using neutron induced autoradiography.

Obtained results are in sharp contrast with the corresponding values for Leggett's biokinetic skeletal model recommended by ICRP, especially in case of cortical bone. According to main results of the study, the authors suggest adjusting the parameters of ICRP biokinetic model and to revise dose coefficients for radiation protection purposes.

*Corresponding author: romanov@subi.su 